\title{
Reliable navigation registration in cranial and spine surgery based on intraoperative computed tomography
}

\author{
Barbara Carl, MD, PhD, ${ }^{1}$ Miriam Bopp, MD,, Benjamin Saß, MD, ${ }^{1}$ Mirza Pojskic, MD, ${ }^{1}$ \\ Marko Gjorgjevski, MD, ${ }^{1}$ Benjamin Voellger, MD, ${ }^{1}$ and Christopher Nimsky, MD, PhD ${ }^{1,2}$ \\ 1Department of Neurosurgery, University of Marburg; and 2Marburg Center for Mind, Brain and Behavior (MCMBB), Marburg, \\ Germany
}

OBJECTIVE Low registration errors are an important prerequisite for reliable navigation, independent of its use in cranial or spinal surgery. Regardless of whether navigation is used for trajectory alignment in biopsy or implant procedures, or for sophisticated augmented reality applications, all depend on a correct registration of patient space and image space. In contrast to fiducial, landmark, or surface matching-based registration, the application of intraoperative imaging allows user-independent automatic patient registration, which is less error prone. The authors' aim in this paper was to give an overview of their experience using intraoperative CT (iCT) scanning for automatic registration with a focus on registration accuracy and radiation exposure.

METHODS A total of 645 patients underwent iCT scanning with a 32-slice movable CT scanner in combination with navigation for trajectory alignment in biopsy and implantation procedures $(n=222)$ and for augmented reality $(n=437)$ in cranial and spine procedures (347 craniotomies and 42 transsphenoidal, 56 frameless stereotactic, 59 frame-based stereotactic, and 141 spinal procedures). The target registration error was measured using skin fiducials that were not part of the registration procedure. The effective dose was calculated by multiplying the dose length product with conversion factors.

RESULTS Among all 1281 iCT scans obtained, 1172 were used for automatic patient registration (645 initial registration scans and 527 repeat iCT scans). The overall mean target registration error was $0.86 \pm 0.38 \mathrm{~mm}( \pm \mathrm{SD}$ ) (craniotomy, $0.88 \pm 0.39 \mathrm{~mm}$; transsphenoidal, $0.92 \pm 0.39 \mathrm{~mm}$; frameless, $0.74 \pm 0.39 \mathrm{~mm}$; frame-based, $0.84 \pm 0.34 \mathrm{~mm}$; and spinal, $0.80 \pm 0.28 \mathrm{~mm}$ ). Compared with standard diagnostic scans, a distinct reduction of the effective dose could be achieved using low-dose protocols for the initial registration scan with mean effective doses of $0.06 \pm 0.04 \mathrm{mSv}$ for cranial, $0.50 \pm 0.09 \mathrm{mSv}$ for cervical, $4.12 \pm 2.13 \mathrm{mSv}$ for thoracic, and $3.37 \pm 0.93 \mathrm{mSv}$ for lumbar scans without impeding registration accuracy.

CONCLUSIONS Reliable automatic patient registration can be achieved using iCT scanning. Low-dose protocols ensured a low radiation exposure for the patient. Low-dose scanning had no negative effect on navigation accuracy.

https://thejns.org/doi/abs/10.3171/2019.8.FOCUS19621

KEYWORDS augmented reality; cranial navigation; effective radiation dose; intraoperative computed tomography; navigation registration; spine navigation

$\mathrm{N}$ AVIGATION is widely used in neurosurgical procedures. Its use in cranial and spine surgery is well established. Whether the tip position of a pointer is identified in image space, devices are aligned to a planned trajectory such as in biopsies and electrode and pedicle screw placement, or advanced augmented reality (AR) visualizations are implemented, all share a common basic navigation concept. Independent from how sophisticated navigation is realized, one of the major basic principles is that a correct registration between real space and image space is mandatory. Low registration errors are crucial to achieve reliable navigation.

Classic landmark- or fiducial-based techniques in cranial surgery, as well as registration strategies based on surface matching, available for cranial and spinal procedures, require user interaction in the registration process.

ABBREVIATIONS AR = augmented reality; DBS = deep brain stimulation; $D L P=$ dose length product; ED = effective dose; iCT = intraoperative CT; iMRI = intraoperative MRI; iUS = intraoperative ultrasound; SEEG = stereo-electroencephalography; TRE = target registration error.

SUBMITTED July 28, 2019. ACCEPTED August 26, 2019.

INCLUDE WHEN CITING DOI: 10.3171/2019.8.FOCUS19621. 
Registration errors in fiducial-based registration have been reported to range from 1.4 to $5.8 \mathrm{~mm},{ }^{22}$ which is comparable to a recent study with errors ranging from 0.55 to $4.8 \mathrm{~mm}^{7}$. Skin surface matching in cranial surgery resulted in even worse mean registration errors of $5.3 \mathrm{~mm},{ }^{19}$ while in spine surgery, bone surface matching achieved lower registration errors of $1.1 \pm 0.61 \mathrm{~mm}$, which markedly increased in adjacent levels. ${ }^{32}$ These results were obtained in Sawbones phantom experiments. Higher precision is typical for phantom experiments with a tight controlled setup and optimized user interaction. Even in well-trained teams, such optimal conditions are not the typical situation in clinical reality, where user dependency and patient dependency negatively influence the registration process.

Intraoperative imaging-based registration does not need patient or user interaction, so it can be automated. ${ }^{8,23}$ This offers the possibility of reduced registration errors, which we could confirm with a mean target registration error (TRE) of $0.93 \mathrm{~mm}$ in an initial series analyzing 200 cranial cases performed with intraoperative CT (iCT) support. ${ }^{4}$

The aim of this paper was to summarize our experience using iCT scanning for automatic registration for navigation-based trajectory alignment and AR in different clinical applications such as craniotomies and transsphenoidal, and frameless and frame-based stereotactic procedures as well as spine procedures, focusing on registration accuracy and radiation exposure.

\section{Methods}

From September 2016 to June 2019, 645 patients (347 males, 298 females; mean age $55.8 \pm 17.5$ years $[ \pm \mathrm{SD}]$, range 4-89 years) were investigated using iCT (347 craniotomies and 42 transsphenoidal, 56 frameless and 59 frame-based stereotactic, and 141 spinal procedures). Procedural details are summarized in Table 1. All patients or appropriate family members gave their informed consent for iCT. We obtained ethics approval for prospective archiving of clinical and technical data applying intraoperative imaging and navigation.

\section{Preoperative Imaging and Image Processing}

Preoperative image data were used for anatomical mapping (mapping Elements, Brainlab) to automatically segment a variety of standard structures, such as the ventricular system, brain anatomy, basal ganglia, vertebrae, and vessels. Unique colors were assigned to each object. Additional manual segmentation (Elements SmartBrush, Brainlab) was applied to correct the results of automatic segmentation, as well as to segment target and risk structures (e.g., tumor, cysts, vessels, disc fragment). In cases in which preoperative multimodality data were available, these data were rigidly registered (Elements Image Fusion, Brainlab). Nonlinear image fusion was applied optionally in spine surgery (Elements Spine Curvature Correction, Brainlab). Trajectory tools (Elements Trajectory Planning and Elements Stereotaxy, Brainlab) helped plan trajectory paths for biopsies, stereo-electroencephalography (SEEG), deep brain stimulation (DBS), and pedicle screw insertion. This altogether formed the navigation plan.
TABLE 1. Procedural characteristics, type of surgery, and navigation use

\begin{tabular}{|c|c|c|c|}
\hline & $\begin{array}{l}\text { Total No. } \\
\text { of Patients }\end{array}$ & AR & $\begin{array}{l}\text { Trajectory } \\
\text { Alignment }\end{array}$ \\
\hline \multicolumn{4}{|l|}{ Craniotomy $(n=347)$} \\
\hline Tumor & 273 & 273 & \\
\hline Glioma & 134 & 134 & \\
\hline Metastasis & 47 & 47 & \\
\hline Meningioma & 46 & 46 & \\
\hline Other & 46 & 46 & \\
\hline Epilepsy surgery (resection \& grids) & 37 & 37 & \\
\hline Vascular & 37 & 37 & \\
\hline \multicolumn{4}{|l|}{ Transsphenoidal $(n=42)$} \\
\hline Pituitary adenoma & 31 & 31 & \\
\hline Other & 11 & 11 & \\
\hline \multicolumn{4}{|l|}{ Frameless stereotaxy $(n=56)$} \\
\hline Biopsy & 38 & & 38 \\
\hline Epilepsy surgery (SEEG) & 8 & & 8 \\
\hline Other & 10 & & 10 \\
\hline \multicolumn{4}{|l|}{ Frame-based stereotaxy $(n=59)$} \\
\hline DBS & 48 & & 48 \\
\hline Epilepsy surgery (SEEG) & 6 & & 6 \\
\hline Biopsy & 5 & & 5 \\
\hline \multicolumn{4}{|l|}{ Spinal $(n=141)$} \\
\hline Tumor & 47 & 26 & 29 \\
\hline Extradural & 28 & 9 & 27 \\
\hline Intradural, extramedullary & 14 & 12 & 2 \\
\hline Intradural, intramedullary & 5 & 5 & \\
\hline Non-tumorous lesions & 5 & 5 & \\
\hline Degenerative & 42 & 12 & 34 \\
\hline Trauma & 21 & & 21 \\
\hline Inflammation & 20 & 2 & 20 \\
\hline Deformity & 2 & 2 & \\
\hline Rheumatoid disease & 2 & & 2 \\
\hline Vascular & 2 & 1 & 1 \\
\hline Total & 645 & 437 & 222 \\
\hline
\end{tabular}

\section{Intraoperative Registration Scanning}

For iCT scanning, a 32-slice movable CT scanner (AIRO, Brainlab) was used. It was closely integrated in a navigation setup consisting of a ceiling-mounted doublemonitor navigation system (Curve, Brainlab) in combination with a dual display system (Buzz, Brainlab). The details of the setting and the main surgical workflows were previously published (craniotomy ${ }^{4}$ and transsphenoidal, frame-based stereotactic, ${ }^{2}$ and spinal ${ }^{3}$ procedures).

After implementation of low-dose scanning for registration, ${ }^{4}$ further dose reduction was established. Table 2 summarizes the parameters of the standard and the most recent low-dose protocols (sinus- $80 \%$ for cranial, c-spine-70\% for cervical, t-spine-70\% for thoracic, and 1spine-70\% for lumbar scans).

The effective dose (ED) was calculated by multiplying 
TABLE 2. CT scanning protocols

\begin{tabular}{cccccc}
\hline & $\begin{array}{c}\text { Protocol } \\
\text { Name }\end{array}$ & Acquisition & kV & $\begin{array}{c}\text { Standard } \\
\text { mA }\end{array}$ & $\begin{array}{c}\text { CTDI } \\
\text { (mGy) }\end{array}$ \\
\hline \multirow{4}{*}{$\begin{array}{c}\text { Head } \\
\text { protocols }\end{array}$} & Axial & 120 & 170 & 64.74 \\
& Helical & 120 & 240 & 64.74 \\
\cline { 2 - 6 } & C-spine & Helical & 120 & 93 & 18.82 \\
\cline { 2 - 6 } & T-spine & Helical & 120 & $110^{*}$ & 22.26 \\
\hline \multirow{nyyyyy}{*}{ L-spine } & Helical & 120 & $110^{*}$ & 22.26 \\
\hline Low-dose & Sinus-80\% & Axial & 120 & 7.07 & 2.70 \\
\cline { 2 - 6 } & Helical & 120 & 10 & 2.70 \\
\cline { 2 - 6 } & C-spine-70\% & Helical & 120 & 28 & 5.66 \\
\cline { 2 - 6 } & T-spine-70\% & Helical & 120 & $33^{*}$ & 6.68 \\
\cline { 2 - 6 } & L-spine-70\% & Helical & 120 & $33^{*}$ & 6.68 \\
\hline
\end{tabular}

$\mathrm{C}=$ cervical; $\mathrm{CTDI}_{\mathrm{vol}}=\mathrm{CT}$ dose index; $\mathrm{L}=$ lumbar; $\mathrm{T}=$ thoracic.

* For these helical scans, weight modulation could be applied (standard weight: $70 \mathrm{~kg}$; adapted $\mathrm{mA}=$ standard $\mathrm{mA} \times$ patient weight/standard patient weight).

the total dose length product (DLP) referring to a phantom with a diameter of $16 \mathrm{~cm}$ (head and cervical) or 32 $\mathrm{cm}$ (thoracic and lumbar) by an ED/DLP conversion factor (head, $2.4 \mu \mathrm{Sv} / \mathrm{mGy} \cdot \mathrm{cm}$; cervical, $5.4 \mu \mathrm{Sv} / \mathrm{Gy} \cdot \mathrm{cm}$; thoracic, $17.8 \mu \mathrm{Sv} / \mathrm{Gy} \cdot \mathrm{cm}$; and lumbar, $19.8 \mu \mathrm{Sv} / \mathrm{Gy} \cdot \mathrm{cm}),{ }^{12,13}$ which, in spine scanning if necessary, was weighted according to the number of vertebrae covered in the cervicothoracic or thoracolumbar junction. If a scout scan was performed to align the scan range of the actual iCT scan, the ED of the scout scan and the ED of the iCT scan were added to calculate the ED of the registration scan.

\section{Navigation Setting}

After completion of the initial iCT scanning, the image data were automatically pushed to the navigation system and automatic patient registration was performed, after which pointer-based navigation was instantaneously available. The TRE was calculated by measuring the offset of 3 skin fiducials that were not part of the registration process.
After completion of this step, the navigation plan was rigidly registered with the $\mathrm{iCT}$ image data (image fusion element); in indicated spine surgery cases, nonlinear registration was applied (see above). In case of repeat iCT scanning and continued navigation usage, the subsequent iCT scans were also scanned with the automatic registration setting.

For trajectory navigation, either dedicated tracked instruments or tracked guide tubes were applied; for the stereotactic frame-based setting, navigation-supported stereotaxy was implemented. ${ }^{2}$ For AR in cranial ${ }^{1,7}$ and spine ${ }^{6}$ surgery, the head-up displays of the operating microscope (Pentero, Pentero 900, and Kinevo 900; all Zeiss) were used.

\section{Results}

A total of 1281 iCT scans were obtained (Table 3). Among them, 1172 iCT scans were used for automatic patient registration, including 645 initial registration scans and 527 repeat iCT scans, which were also used for registration and continued navigation. Automatic registration was performed on 891 cranial iCT scans and 281 spinal iCT scans. Automatic registration was used for 222 trajectory alignment procedures in biopsies and implantation procedures (56 frameless stereotaxy, 59 frame-based stereotaxy, and 107 spinal implant procedures [instrumentation]) and for 437 AR applications in cranial and spine procedures ( 347 craniotomies and 42 transsphenoidal and 48 spinal procedures; Table 3 ).

\section{Registration Accuracy}

The mean TRE for the initial registration scan of all procedures was $0.86 \pm 0.38 \mathrm{~mm}$ (range $0.05-2.19 \mathrm{~mm}$ ). Analyzing the TRE according to the 5 categories resulted in the following mean TREs: craniotomies, $0.88 \pm 0.39$ $\mathrm{mm}$ (range $0.05-2.19 \mathrm{~mm}$ ); transsphenoidal procedures, $0.92 \pm 0.39 \mathrm{~mm}$ (range 0.21-2.07 mm); stereotaxy (frameless) procedures, $0.74 \pm 0.39 \mathrm{~mm}$ (range $0.12-1.86 \mathrm{~mm}$ ); stereotaxy (frame-based), $0.84 \pm 0.34 \mathrm{~mm}$ (range $0.30-1.74$ $\mathrm{mm}$ ); and spinal procedures, $0.80 \pm 0.28 \mathrm{~mm}$ (range $0.41-$ $1.45 \mathrm{~mm}$ ). Comparing the different subgroups showed no

TABLE 3. Total number of iCT scans obtained classified according to different procedural categories

\begin{tabular}{ccccccc}
\hline \multirow{2}{*}{$\begin{array}{c}\text { No. of iCT } \\
\text { Scans/Case }\end{array}$} & Craniotomy & Transsphenoidal & Frameless & Frame-Based & Spinal & Total \\
& $87(87 / 87)$ & $31(31 / 31)$ & $12(12 / 12)$ & & $40(40 / 40)$ & $170(170 / 170)$ \\
\hline 1 & $253(506 / 506)$ & $11(18 / 22)$ & $42(53 / 84)$ & $21(29 / 42)$ & $69(136 / 138)$ & $396(742 / 792)$ \\
\hline 3 & $7(21 / 21)$ & & $1(3 / 3)$ & $5(12 / 15)$ & $19(49 / 57)$ & $32(85 / 96)$ \\
\hline 4 & & $1(4 / 4)$ & $19(55 / 76)$ & $6(22 / 24)$ & $26(81 / 104)$ \\
\hline 5 & & & $8(31 / 40)$ & $4(16 / 20)$ & $12(47 / 60)$ \\
\hline 7 & & & $4(19 / 24)$ & $1(5 / 6)$ & $5(24 / 30)$ \\
\hline 8 & & & $2(10 / 14)$ & $1(7 / 7)$ & $3(17 / 21)$ \\
\hline Total & $347(614 / 614)$ & $42(49 / 53)$ & $56(72 / 103)$ & $59(156 / 211)$ & $141(281 / 300)$ & $645(1172 / 1281)$ \\
\hline
\end{tabular}

A total of 1281 iCTs were obtained in 645 patients, among which 1172 were used for automatic registration. Data are presented as the number of cases (number of iCT scans used for automatic registration/total number of iCT scans). 


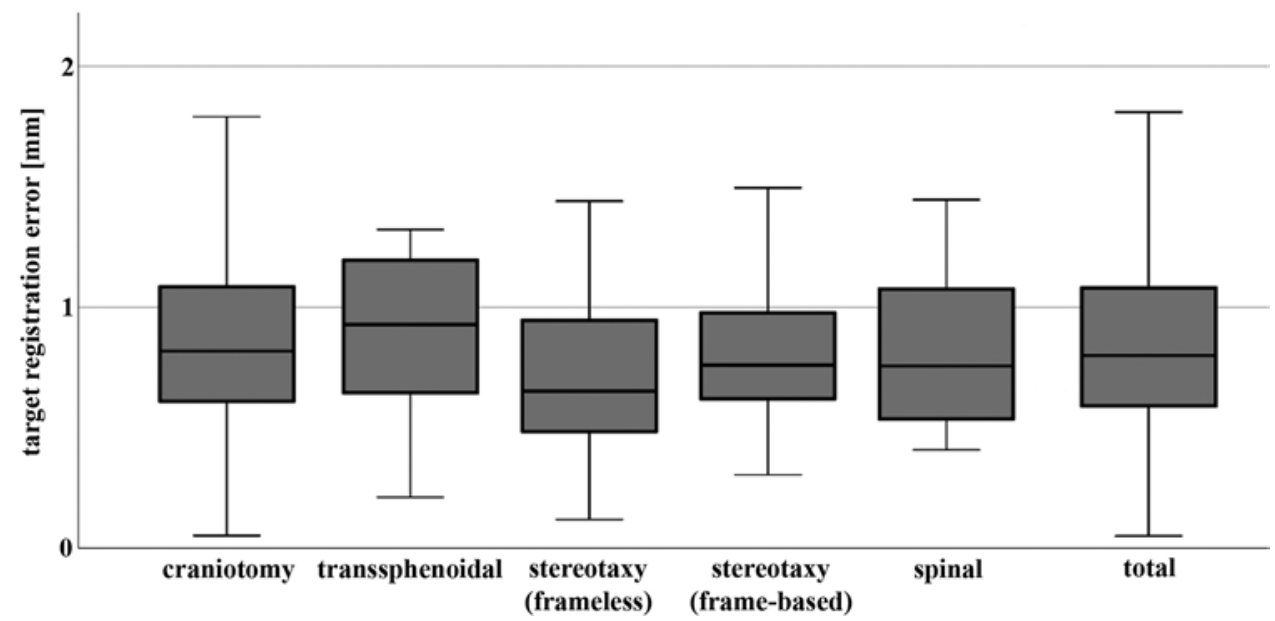

FIG. 1. Tukey box plot of the TRE classified by different surgical categories. The horizontal line and box represent the median and $I Q R$, respectively. The whiskers represent the range with outliers excluded.

significant differences; a box plot visualizing these data is shown in Fig. 1. Figure 2 depicts the TRE grouped according to the different scanning protocols, showing that dose reduction did not significantly influence the TRE. Figure 3 demonstrates how the registration accuracy is checked with the navigation pointer and the operating microscope, identifying the position of a skin fiducial that was not part of the registration process.

Additionally, to determine the TRE, the registration accuracy was checked by identifying anatomical landmarks, as well as artificial landmarks (e.g., inserted retractor systems in spine surgery). For repeat iCT scanning, registration accuracy was checked in the spine procedures using the skin fiducial landmarks, the retractor systems, and bone edges, all proving reliable navigation registration. In the craniotomy procedures, the identification of the x-ray contrast filament of a cotton patty placed in the resection cavity in updated navigation proved a low registration error in all cases. AR was used in a frame-based stereotactic procedure to visually demonstrate that the entire registra- tion pipeline, including patient registration and AR calibration, had a very low overall error by showing the close matching of the segmented surface object, including the frame localizer and the patient's skin, with reality (Fig. 4). In cases in which implants were inserted in spine surgery, especially vertebral body replacements, repeat iCT scanning to check the implant position could be used for updating navigation. Segmentation of the implant and its subsequent visualization with updated AR allowed not only patient accuracy to be checked but also the overall registration pipeline, including patient registration, image registration, and AR calibration (Fig. 5).

\section{Effective Radiation Dose}

The mean ED for the initial registration scan applying the latest low-dose protocols (sinus-80\%, c-spine-70\%, tspine-70\%, and 1-spine-70\%; Table 2), including the scout scan, was as follows: cranial region, $0.06 \pm 0.04 \mathrm{mSv}$ (range 0.02-0.19 mSv); cervical region, $0.50 \pm 0.09 \mathrm{mSv}$

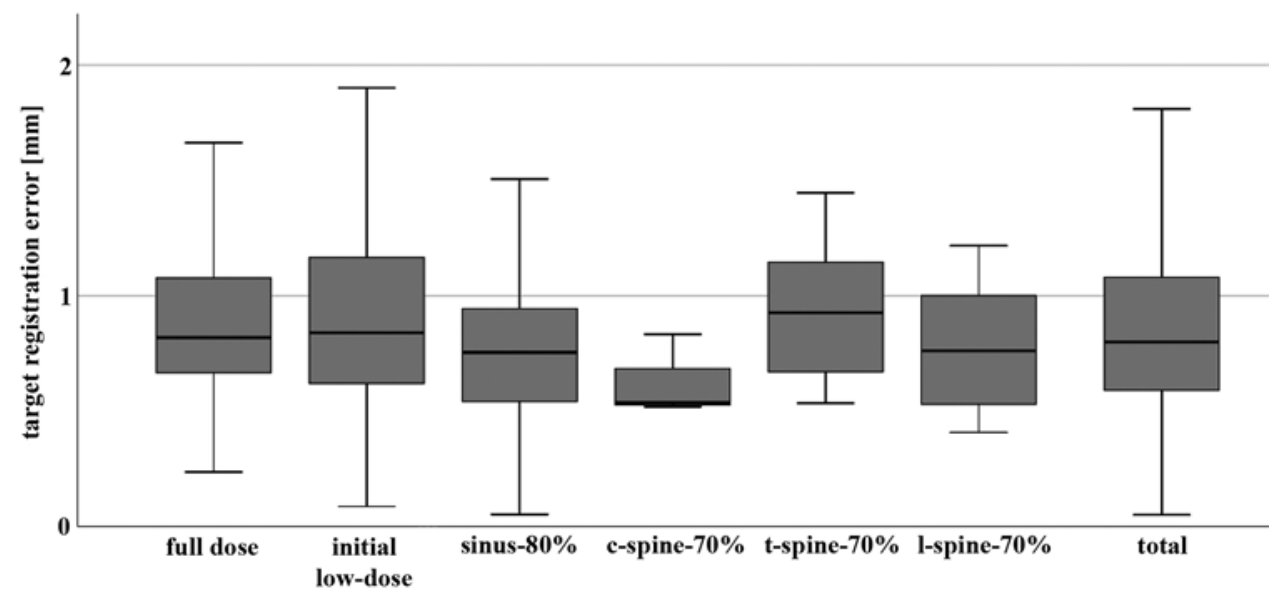

FIG. 2. Tukey box plot of the TRE classified by different registration scan protocols (initial low-dose protocols). $c=$ cervical; I = lumbar; $\mathrm{t}=$ thoracic. 


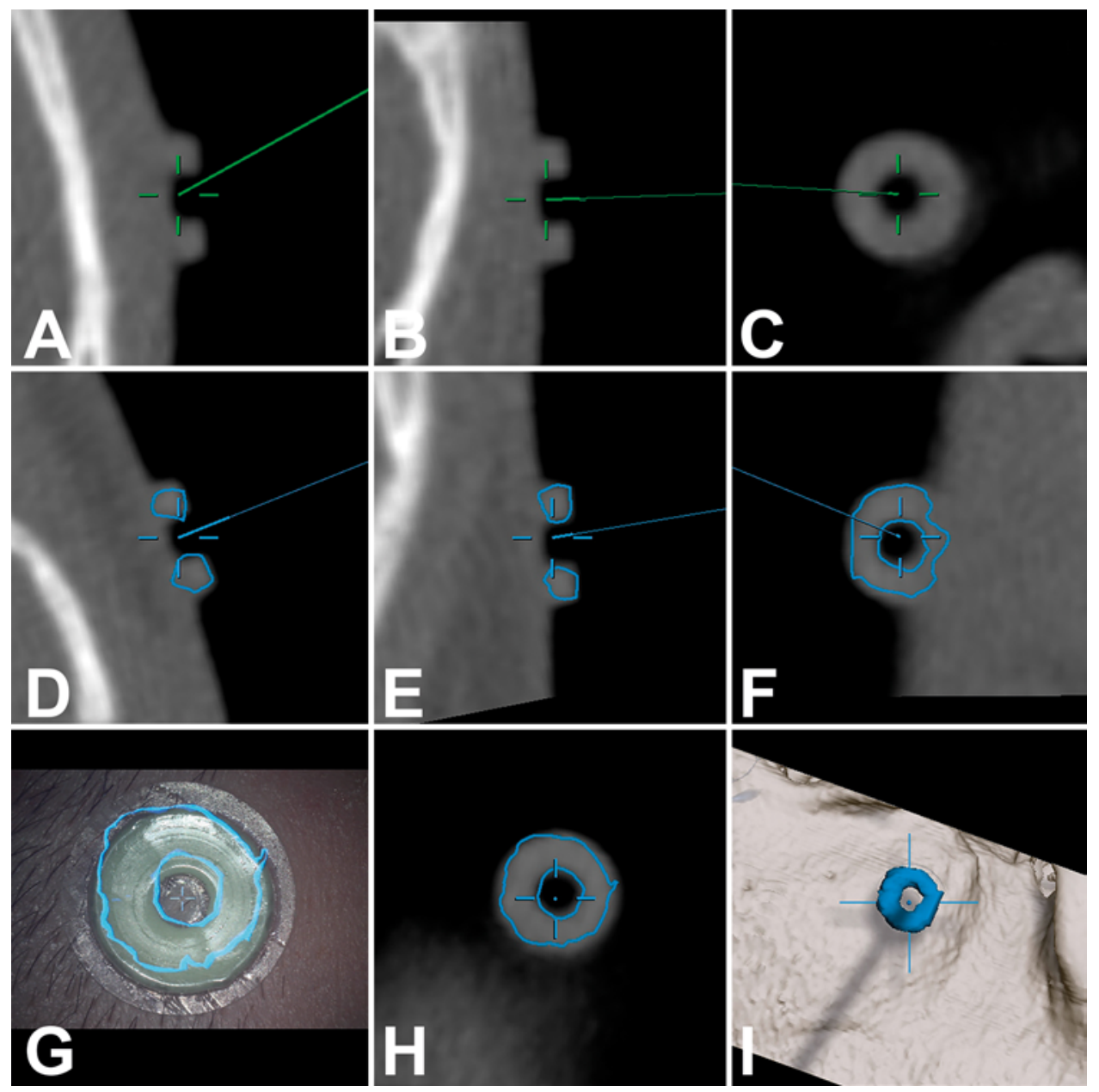

FIG. 3. Checking registration accuracy by placing the tip of the navigation pointer in the center of a skin fiducial. A-C: Axial (A), coronal (B), and sagittal (C) images. The tip of the pointer and the pointer alignment in 3D space are visualized as a green crosshair and green line, respectively. D-I: Axial (D), coronal (E), and sagittal (F) images. When focusing with the operating microscope in the same position, the microscope autofocus center and alignment in 3D space are visualized in blue. Additionally, the fiducial was segmented in blue (D-I), so that the close matching of the segmented fiducial object and reality could be confirmed by AR (G). Corresponding probe's-eye views in $2 \mathrm{D}(\mathrm{H})$ and $3 \mathrm{D}(\mathrm{I})$.

(range 0.31-0.69 mSv); thoracic region, $4.12 \pm 2.13 \mathrm{mSv}$ (range 1.48-9.64 $\mathrm{mSv}$ ); and lumbar region, $3.37 \pm 0.93$ $\mathrm{mSv}$ (range 1.59-5.01 mSv).

\section{Discussion}

\section{Navigation Registration}

Accurate registration is a prerequisite for reliable navigation, independent of its use to align an instrument in 3D space to a preplanned trajectory such as in biopsy and implantation procedures, to delineate a position in 3D space with the tip of a pointer, or to enable advanced AR visualizations applying the head-up displays of operating microscopes.

Except for frame-based stereotactic procedures, where the registration process is user independent because the known geometry of the localizer box is scanned either pre- or intraoperatively, in all other cranial and spinal procedures, registration typically involves some user interaction. For registration in cranial procedures (e.g., anatomi- cal landmarks have to be assigned to their corresponding positions in the 3D image data or skin fiducials have to be localized with a tracked pointer and assigned to their corresponding positions in the 3D image data), alternatively surface scanning can be applied.

The most common method for registration is the application of adhesive skin fiducials, which provide an additional factor of uncertainty; these markers are usually placed on the patient's skin on the day prior to surgery, and their position might move during the time of imaging and actual registration. Furthermore, the pattern of how the fiducials are arranged on the skin influences the registration accuracy; however, even if an optimized spatial placement method is applied, TREs in the range of $3 \mathrm{~mm}$ can result. ${ }^{25}$ Phantom experiments might report reliable registration; however, all these factors explain easily why in standard clinical settings quite variable TREs in the range of 1.4 to $5.8 \mathrm{~mm}^{22}$ or 0.55 to $4.8 \mathrm{~mm}^{7}$ are reported. A recent study analyzing the effect of additional skin surface matching showed a worsening of registration accuracy with an in- 

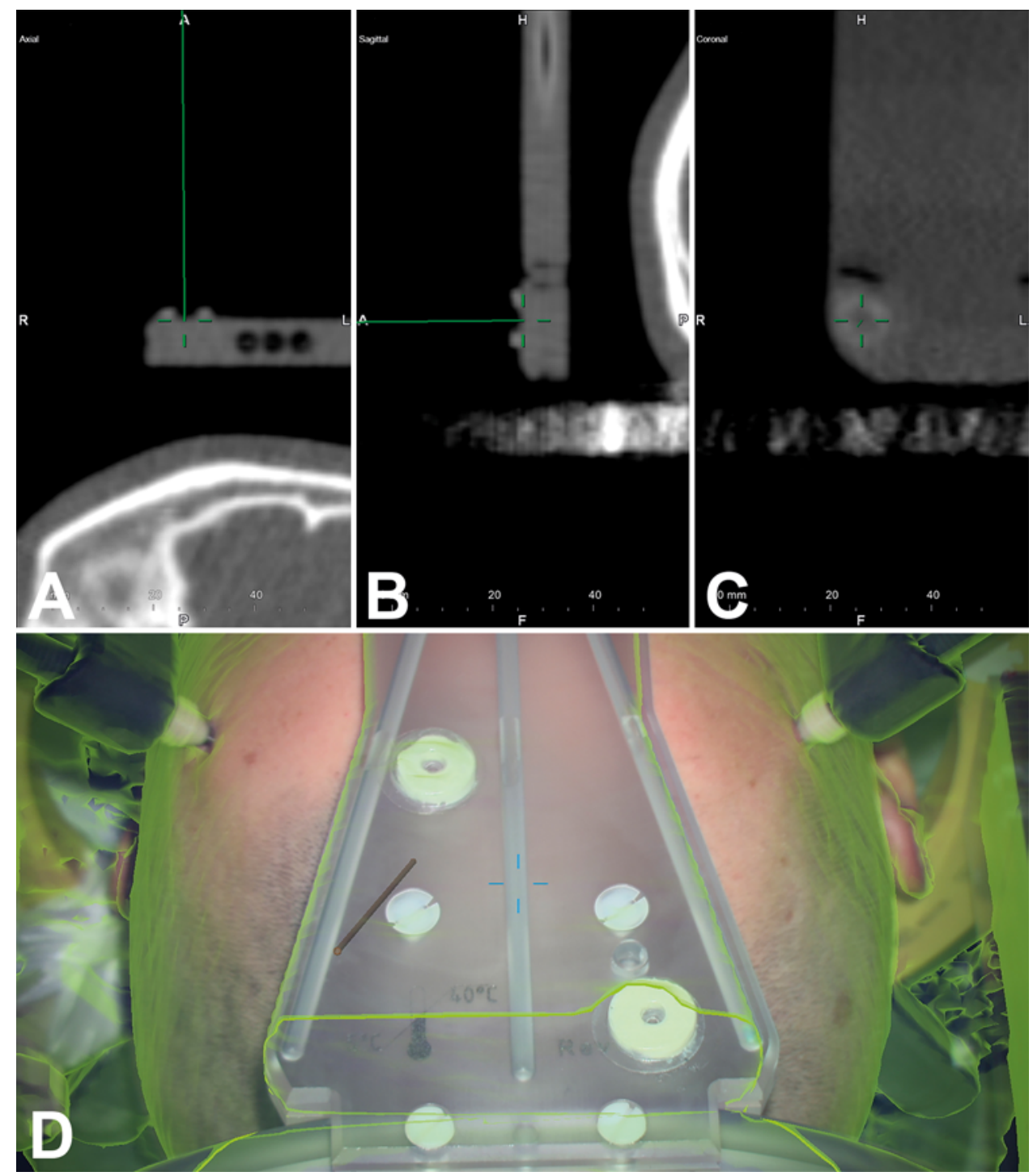

FIG. 4. Case example of navigation-supported frame-based stereotaxy. Skin fiducials are placed on the stereotactic localizer so that their positions can be identified with a navigation pointer. A-C: Axial (A), sagittal (B), and coronal (C) images. The tip of the pointer and the pointer alignment in 3D space are visualized as a green crosshair and green line, respectively. D: To visualize the low registration error, AR was applied in this case for visualization, depicting a very close matching of the surface object with reality (light green; the surface object includes the stereotactic localizer and the skin of the patient).

crease in the mean registration errors from $5.3 \mathrm{~mm}$ to 6.3 mm. ${ }^{19}$

While in cranial surgery skin surface matching leads only to mediocre registration accuracy, in spine surgery for a single vertebra surface matching-based registration is an established strategy for spinal implantation of pedicle screws. This is due to the direct identification of the bony surface by sampling at least 20 points on the lamina of a vertebra with the tip of a navigation pointer, ${ }^{28}$ which in phantom measurements achieves low registration errors of $1.1 \pm 0.61 \mathrm{~mm}$ but markedly increases in adjacent levels. ${ }^{32}$ This strategy is only clinically applicable in posterior open spine surgeries; if multiple levels are operated, repeated registration has to be performed for each vertebra. In spine procedures, fiducial-based registration ${ }^{29}$ is only an option when intraoperative imaging is available, because the flexibility of the spine more or less excludes that the alignment of the vertebra during preoperative registration scanning is identical to the intraoperative alignment on the operating table.

\section{Automatic Registration}

User-independent registration can be achieved by intraoperative imaging. In regard to spine surgery, this has the additional advantage in that patient positioning and therefore vertebral alignment during imaging corresponds to the real intraoperative situation. There are different concepts of how automatic registration based on intraoperative imaging can be realized. A solution is to place a tracked object in image space, so that by knowing its pre- 

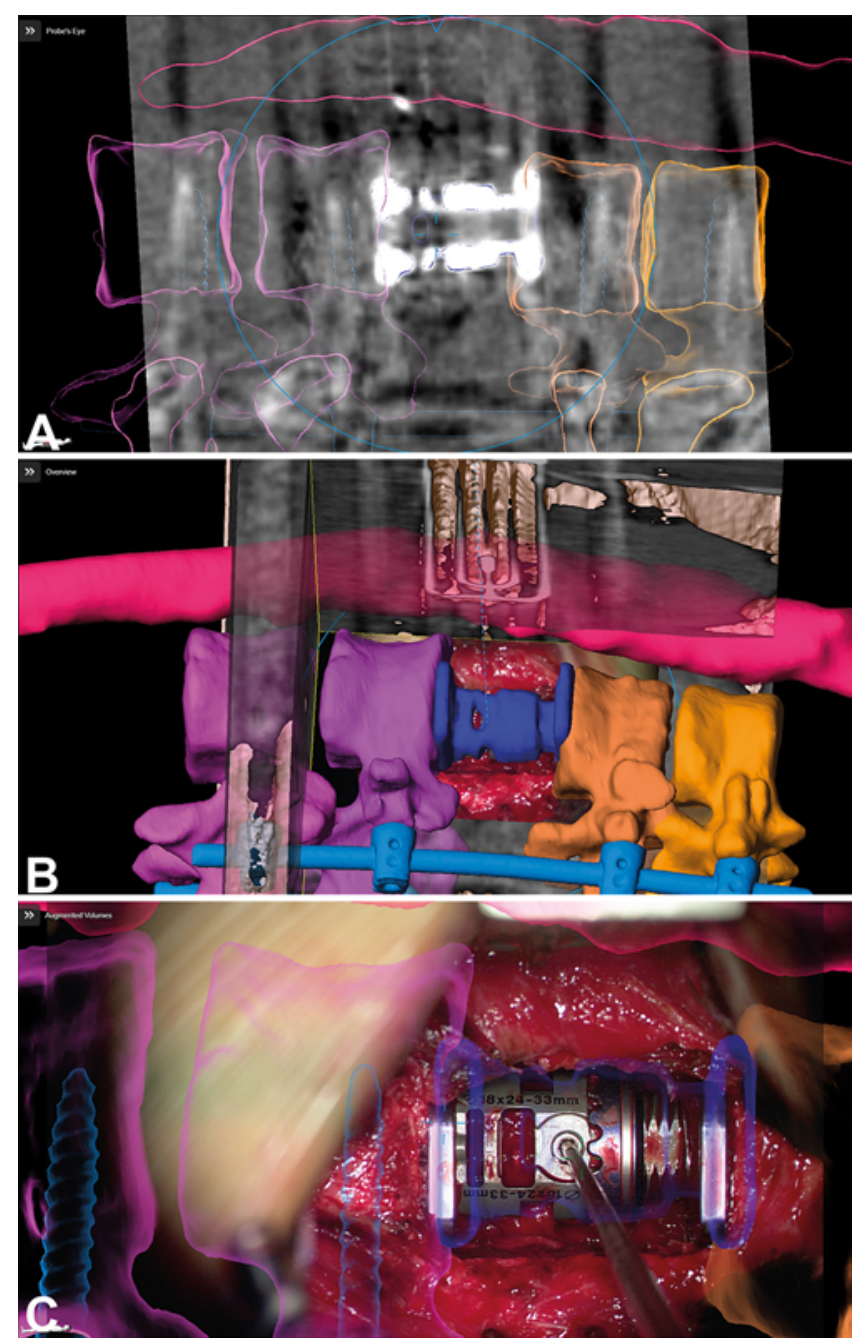

FIG. 5. Images obtained in a patient with an osteoclastoma at L1. Vertebral body replacement was performed with an expandable implant; repeat iCT confirmed the correct implant position, the implant was segmented in dark blue, navigation was updated with the repeat iCT data, and the AR visualization of the implant matched with reality very closely, demonstrating that the entire registration pipeline including patient registration, image fusion, and AR calibration was highly accurate. A: Probe's-eye view. B: Overview 3D visualization of the segmented objects, depicting the position of the microscope video frame. C: AR view depicting the close matching of the implant and the AR object representing the implant.

cise geometry it can be automatically detected in the 3D image data, allowing definition of the transformation of the image coordinate system to the real physical patient space. This is the typical setting of how automatic registration is implemented in intraoperative MRI (iMRI). ${ }^{23}$ Alternatively, the imaging space of the scanner is calibrated to the navigation system, such that it is prototypically implemented in a low-field iMRI system ${ }^{16}$ or is enabled by tracking the scanner during imaging, as it was first established in a sliding gantry iCT concept, resulting in TREs below $2 \mathrm{~mm} .{ }^{8,9}$

We had implemented this concept of user-independent automatic registration applying iCT scanning and showed a low mean TRE of $0.93 \mathrm{~mm}$ in an initial series analyzing 200 cranial surgeries, including 141 craniotomies, 19 transsphenoidal procedures, and 40 burr hole procedures. ${ }^{4}$ Our current series confirms these initial findings with an overall mean TRE of $0.86 \mathrm{~mm}$, which did not show any significant difference between the 5 investigated categories: craniotomy and transsphenoidal, frameless and frame-based stereotactic, and spinal procedures.

Our results for spine surgery with a mean TRE of 0.80 $\pm 0.28 \mathrm{~mm}$ correspond well to the results of a phantom study for spine registration that compared automatic intraoperative imaging-based registration and bone surface registration $(0.74 \mathrm{~mm}$ vs $1.1 \mathrm{~mm}),{ }^{32}$ as well as to a spine phantom experiment using iCT and bone-implanted miniscrews with a TRE that ranged from 0.14 to $0.78 \mathrm{~mm} .^{14}$

\section{Effective Radiation Dose}

The principal drawback of iCT-based automatic registration is that it means that the patient is exposed to radiation. Low-dose protocols allowed for significant reduction of the ED. The mean ED applying low-dose protocols for the registration scans was $0.06 \mathrm{mSv}$ for cranial, $0.50 \mathrm{mSv}$ for cervical, $4.12 \mathrm{mSv}$ for thoracic, and $3.37 \mathrm{mSv}$ for lumbar scans, meaning a reduction by a factor of more than 30 for cranial, and a factor between 2.5 and 6 for the spinal scans in relation to comparable standard diagnostic scans. ${ }^{10,17,26,27,30}$ The major reasons higher dosages are necessary in spine imaging are that the scanned body volume is much larger than that of the head due to the anatomy, with an increased diameter of the thorax and abdomen in comparison to the head, and the scan ranges are longer than in cranial registration imaging. Especially in the midthoracic region, relatively long scan ranges are required to ensure unambiguous vertebra assignment and reliable nonlinear registration with the preoperative images. Furthermore, tissue weighting factors increase the calculated ED for spine scans.

Despite significant dose reduction we did not observe an increase of the registration error, which otherwise would mean a decreased navigation accuracy. The current low-dose protocols have not yet reached the principle limit at which artifacts from image noise and decreased resolution prevent reliable identification of structures that can be used for fusion of the registration images with highquality data sets. ${ }^{11,24}$ Phantom experiments suggest that these fundamental limits might be reached at $1 \mathrm{mAs} .{ }^{15} \mathrm{In}$ spine registration, metallic implants that were previously inserted, like in previous spine instrumentation, might provide enough structural information for subsequent image registration, so that the application of very low-dose protocols might be possible, as in neonate protocols with a decreased scan voltage of $80 \mathrm{kV} .^{5}$

For cranial registration, we already have achieved a very low $\mathrm{ED}$ for a single registration scan, which is only one-fiftieth of the typical individual exposure of radiation of a US citizen from natural background sources of about $3 \mathrm{mSv}$ per year.

\section{Radiation-Free Automatic Registration Alternatives to iCT}

For cranial surgery, iMRI is well established with the possibility of automatic registration applying a reference 
array to the imaging coil whose geometrical structure can be detected automatically on the intraoperative images, enabling automatic registration. In phantom studies, this resulted in TREs between 0.88 and $2.13 \mathrm{~mm}$, which was a significantly better registration than the standard fiducialbased registration in this study. ${ }^{23}$ However, from these data it cannot be concluded that automatic iCT registration is more accurate than automatic iMRI registration. Unfortunately, more data investigating the automatic registration accuracy of applying iMRI in clinical reality that would allow a comparison with the clinical iCT registration have not been published. CT-based registration per se has the benefit that the images are not geometrically distorted, which is an inherent problem of some MR sequences. However, considering MR data integration in navigation when using iCT-based registration, this MR image distortion has also to be taken into account, as well as the image registration process itself, which poses an additional potential source for registration inaccuracies.

In spine surgery, registration using iMRI is at the moment only possible with the application of fiducials, as demonstrated in a series of patients undergoing interstitial laser thermotherapy and iMRI; ${ }^{29}$ this setting, however, does not provide the possibility of automatic registration, so that the registration errors are expected to be significantly higher than in automatic iCT-based registration in spinal procedures.

In spine surgery, there have been some experimental attempts to use intraoperative ultrasound (iUS) for registration of the bone surface structures to reconstruct the bone geometry. However, these setups are still in an early experimental state and not yet accurate enough for clinical application. ${ }^{18,20,31}$ In cranial surgery, iUS might be applied for navigation registration in craniotomy procedures; however, this is not applicable for transsphenoidal or burr hole procedures such as frameless or frame-based stereotactic procedures. In craniotomies, the actual patient registration might be performed directly by iUS imaging, which then defines the actual navigation coordinate system and allows subsequent integration of preoperative images by image registration of preoperative images with the 3D-iUS data. Such an image registration based on 3D ultrasound images is still challenging and might need additional nonlinear components; nevertheless, in a recent study an initial mean TRE measuring the image registration accuracy could be reduced from 16.9 to $3.8 \mathrm{~mm}$ using an intensity-based registration and to $2.2 \mathrm{~mm}$ with a combined segmentation and registration approach. ${ }^{21}$

Of course, one might ask whether registration errors, as low as we achieved with the automatic iCT-based registration of less than $1 \mathrm{~mm}$, are necessary at all in different kinds of surgery, justifying the effort of intraoperative imaging in general and, in case of $\mathrm{iCT}$, the radiation exposure for the patient in particular. Since a reliable registration is probably the most important, but not only, step in the entire pipeline of registration, it is crucial to minimize each factor contributing to inaccuracy. When implementing AR based on multimodality image data, image registration errors and calibration errors also contribute to the overall accuracy of the AR visualization. High precision is needed if electrodes are implanted for DBS or SEEG, as well as in biopsies in eloquent regions. In spine surgery, the flexibility of the spine contributes to potentially distinct errors if unsophisticated nonlinear image registration approaches are used to compensate for the spatial flexibility, necessitating low initial patient registration errors and accurate information on the actual 3D configuration of the spine during surgery, both of which can be reliably provided only by intraoperative imaging.

Automatic registration is only one, but an important, aspect for justifying the use of intraoperative imaging. Intraoperative imaging allows for a re-registration either if an unwanted event results in a positional shifting (e.g., movement of the reference array in relation to the surgical site occurred), or in cases in which surgical alterations (e.g., removal of a tumor) result in a significant brain shift, making navigation no longer reliably usable; these phenomena of course also apply for spine navigation.

Additionally, intraoperative imaging provides an immediate intraoperative quality control with the possibility to avoid complications and evaluate whether the aim of surgery was met, i.e., to provide information on the extent of a tumor resection, whether the intended target was biopsied, or whether electrodes or other implants are correctly placed according to plan, avoiding unwanted surprises on postoperative imaging that might necessitate reoperations, which are beyond the analysis of $\mathrm{ICT}$ in this paper. Intraoperative imaging can identify an incomplete resection; residual tumor portions that were initially missed can be removed during the same operation without much guesswork. Updated navigation facilitates localizing these tumor remnants. In contrast to the subjective surgeon's estimate, intraoperative imaging allows an objective evaluation of the intraoperative situation, thus acting as quality control during surgery.

\section{Conclusions}

Reliable automatic patient registration can be achieved using iCT scanning. Low-dose protocols ensured a low radiation exposure for the patient. Low-dose scanning had no negative effect on navigation accuracy.

\section{References}

1. Carl B, Bopp M, Chehab S, Bien S, Nimsky C: Preoperative 3-dimensional angiography data and intraoperative real-time vascular data integrated in microscope-based navigation by automatic patient registration applying intraoperative computed tomography. World Neurosurg 113:e414-e425, 2018

2. Carl B, Bopp M, Gjorgjevski M, Nimsky C: Navigationsupported stereotaxy by applying intraoperative computed tomography. World Neurosurg 118:e584-e592, 2018

3. Carl B, Bopp M, Pojskic M, Voellger B, Nimsky C: Standard navigation versus intraoperative computed tomography navigation in upper cervical spine trauma. Int J CARS 14:169182,2019

4. Carl B, Bopp M, Saß B, Nimsky C: Intraoperative computed tomography as reliable navigation registration device in 200 cranial procedures. Acta Neurochir (Wien) 160:1681-1689, 2018

5. Carl B, Bopp M, Sass B, Pojskic M, Voellger B, Nimsky C: Spine surgery supported by augmented reality. Global Spine J [in press], 2019

6. Carl B, Bopp M, Saß B, Voellger B, Nimsky C: Implemen- 
tation of augmented reality support in spine surgery. Eur Spine J 28:1697-1711, 2019

7. Carl B, Bopp M, Voellger B, Saß B, Nimsky C: Augmented reality in transsphenoidal surgery. World Neurosurg 125:e873-e883, 2019

8. Eggers G, Kress B, Mühling J: Fully automated registration of intraoperative computed tomography image data for image-guided craniofacial surgery. J Oral Maxillofac Surg 66:1754-1760, 2008

9. Eggers G, Kress B, Rohde S, Mühling J: Intraoperative computed tomography and automated registration for imageguided cranial surgery. Dentomaxillofac Radiol 38:28-33, 2009

10. Fazel R, Krumholz HM, Wang Y, Ross JS, Chen J, Ting HH, et al: Exposure to low-dose ionizing radiation from medical imaging procedures. N Engl J Med 361:849-857, 2009

11. Greffier J, Pereira FR, Viala P, Macri F, Beregi JP, Larbi A: Interventional spine procedures under $\mathrm{CT}$ guidance: how to reduce patient radiation dose without compromising the successful outcome of the procedure? Phys Med 35:88-96, 2017

12. Huda W, Magill D, He W: CT effective dose per dose length product using ICRP 103 weighting factors. Med Phys 38:1261-1265, 2011

13. Huda W, Ogden KM, Khorasani MR: Converting dose-length product to effective dose at CT. Radiology 248:995-1003, 2008

14. Ji S, Fan X, Paulsen KD, Roberts DW, Mirza SK, Lollis SS: Intraoperative $\mathrm{CT}$ as a registration benchmark for intervertebral motion compensation in image-guided open spinal surgery. Int J CARS 10:2009-2020, 2015

15. Ketcha MD, de Silva T, Han R, Uneri A, Goerres J, Jacobson M, et al: Fundamental limits of image registration performance: effects of image noise and resolution in CT-guided interventions. Proc SPIE Int Soc Opt Eng 10135:1013508, 2017

16. Lewin JS, Petersilge CA, Hatem SF, Duerk JL, Lenz G, Clampitt ME, et al: Interactive MR imaging-guided biopsy and aspiration with a modified clinical C-arm system. AJR Am J Roentgenol 170:1593-1601, 1998

17. Lin EC: Radiation risk from medical imaging. Mayo Clin Proc 85:1142-1146, 2010

18. Ma L, Zhao Z, Chen F, Zhang B, Fu L, Liao H: Augmented reality surgical navigation with ultrasound-assisted registration for pedicle screw placement: a pilot study. Int J CARS 12:2205-2215, 2017

19. Mongen MA, Willems PWA: Current accuracy of surface matching compared to adhesive markers in patient-to-image registration. Acta Neurochir (Wien) 161:865-870, 2019

20. Nagpal S, Abolmaesumi P, Rasoulian A, Hacihaliloglu I, Ungi T, Osborn J, et al: A multi-vertebrae CT to US registration of the lumbar spine in clinical data. Int J CARS 10:1371-1381, 2015

21. Nitsch J, Klein J, Dammann P, Wrede K, Gembruch O, Moltz $\mathrm{JH}$, et al: Automatic and efficient MRI-US segmentations for improving intraoperative image fusion in image-guided neurosurgery. Neuroimage Clin 22:101766, 2019

22. Pfisterer WK, Papadopoulos S, Drumm DA, Smith K, Preul MC: Fiducial versus nonfiducial neuronavigation registration assessment and considerations of accuracy. Neurosurgery 62 (3 Suppl 1):201-208, 2008
23. Rachinger J, von Keller B, Ganslandt O, Fahlbusch R, Nimsky C: Application accuracy of automatic registration in frameless stereotaxy. Stereotact Funct Neurosurg 84:109_ 117,2006

24. Sarwahi V, Payares M, Wendolowski S, Maguire K, Thornhill B, Lo Y, et al: Low-dose radiation 3D intraoperative imaging: how low can we go? An O-arm, CT scan, cadaveric study. Spine (Phila Pa 1976) 42:E1311-E1317, 2017

25. Shamir RR, Joskowicz L, Shoshan Y: Fiducial optimization for minimal target registration error in image-guided neurosurgery. IEEE Trans Med Imaging 31:725-737, 2012

26. Smith-Bindman R, Lipson J, Marcus R, Kim KP, Mahesh M, Gould R, et al: Radiation dose associated with common computed tomography examinations and the associated lifetime attributable risk of cancer. Arch Intern Med 169:20782086, 2009

27. Smith-Bindman R, Moghadassi M, Wilson N, Nelson TR, Boone JM, Cagnon $\mathrm{CH}$, et al: Radiation doses in consecutive CT examinations from five University of California medical centers. Radiology 277:134-141, 2015

28. Tamura Y, Sugano N, Sasama T, Sato Y, Tamura S, Yonenobu $\mathrm{K}$, et al: Surface-based registration accuracy of CT-based image-guided spine surgery. Eur Spine J 14:291-297, 2005

29. Tatsui CE, Nascimento CNG, Suki D, Amini B, Li J, Ghia AJ, et al: Image guidance based on MRI for spinal interstitial laser thermotherapy: technical aspects and accuracy. J Neurosurg Spine 26:605-612, 2017

30. Vilar-Palop J, Vilar J, Hernández-Aguado I, González-Álvarez I, Lumbreras B: Updated effective doses in radiology. J Radiol Prot 36:975-990, 2016

31. Winter S, Pechlivanis I, Dekomien C, Igel C, Schmieder $\mathrm{K}$ : Toward registration of 3D ultrasound and CT images of the spine in clinical praxis: design and evaluation of a data acquisition protocol. Ultrasound Med Biol 35:1773-1782, 2009

32. Zhao J, Liu Y, Fan M, Liu B, He D, Tian W: Comparison of the clinical accuracy between point-to-point registration and auto-registration using an active infrared navigation system. Spine (Phila Pa 1976) 43:E1329-E1333, 2018

\section{Disclosures}

Drs. Carl and Nimsky: speaker honoraria from Brainlab.

\section{Author Contributions}

Conception and design: Carl, Nimsky. Acquisition of data: all authors. Analysis and interpretation of data: Carl, Bopp, Nimsky. Drafting the article: Carl, Bopp, Nimsky. Critically revising the article: Carl, Nimsky. Reviewed submitted version of manuscript: all authors. Approved the final version of the manuscript on behalf of all authors: Carl. Statistical analysis: Bopp. Study supervision: Nimsky.

\section{Correspondence}

Barbara Carl: University of Marburg, Germany. carlb@med. uni-marburg.de. 